\title{
Review
}

\section{Molecular Therapy Using Ultrasound : Mechanisms Involved in Drug Activation, Apoptosis Induction, Gene Transfer, and Alterations of Gene Expression}

\author{
TORU YOSHIDA ${ }^{1}$, TAKASHI KONDO ${ }^{2 *}$, RYOHEI OGAWA ${ }^{2}$, QING-LI ZHAO ${ }^{2}$, \\ MARIAME A. HASSAN ${ }^{2}$, AKIHIKO WATANABE ${ }^{3}$, ICHIRO TAKASAKI ${ }^{4}$, \\ YOSHIAKI TABUCHI ${ }^{4}$, MIKI SHOJI ${ }^{4}$, NOBUKI KUDO ${ }^{5}$, LORETO B. FERIL, JR. ${ }^{6}$, \\ KATSURO TACHIBANA ${ }^{6}$, MIKHAIL A. BULDAKOV ${ }^{7,8}$, TAKASHI HONDA ${ }^{9}$, \\ KAZUHIRO TSUKADA ${ }^{1}$, PETER RIESZ ${ }^{10}$
}

\footnotetext{
${ }^{1}$ Second Department of Surgery, ${ }^{2}$ Department of Radiological Sciences, ${ }^{3}$ Department of Urology, Graduate School of Medicine and Pharmaceutical Sciences, ${ }^{4}$ Division of Molecular Genetics Research, Life Science Research Center, University of Toyama, Sugitani 2630, Toyama 930-0194, Japan

${ }^{5}$ Laboratory of Biomedical Instrumentation and Measurements, Graduate School of Information Science and Technology, Hokkaido University, Kita 14, Nishi 9, Sapporo 060-0814, Japan

${ }^{6}$ Department of Anatomy, Fukuoka University School of Medicine, Nanakuma 7, Johnan, Fukuoka 814-0180, Japan

${ }^{7}$ Cancer Research Institute, Tomsk Scientific Center, Siberian Department of the Russian Academy of Medical Sciences, Kooperativniy Street, Tomsk 634009, Russia

${ }^{8}$ Tomsk State University, Leninskiy prospect, Tomsk 634050, Russia

${ }^{9}$ Kinjo University, Kasama-cho 1200, Hakusan, Ishikawa 924-8511, Japan

${ }^{10}$ Radiation Biology Branch, National Cancer Institute, NIH, 9000 Rockville Pike, Bethesda MD, USA
}

Abstract: Interest in molecular imaging and in molecularly-targeted therapy has grown tremendously, and ultrasound may offer new tools for modern cancer therapy. To understand how therapeutic ultrasound works, it is necessary to understand its biological effects at the molecular level. In this review, investigations on the molecular aspects of ultrasound are discussed, with emphasis on apoptosis induction, gene expression, and gene transfection. In these studies, apoptosis induction was assayed with flow cytometry and with other methods targeting indicators of apoptosis. Gene expression was evaluated using western blotting, real-time polymerase chain reaction, and microarray analysis. Gene transfection was investigated using a luciferase assay and other methods. The reported results show that low intensity ultrasound can induce apoptosis in cancer cell lines, and that this effect can be optimized using pulsed ultrasound. Exposure to ultrasound can result in the down-regulation or up-regulation of some genes. Of particular interest is the striking up-regulation of the heme oxygenase-1 gene, a gene usually associated with oxidative stress in human lymphoma U937 cells. Introducing genes using

Received 3 July 2007, Accepted 20 August 2007. ${ }^{*}$ Corresponding author ; Tel, +81-76-434-7265; Fax, +81-76-434-5190 ;

e-mail,kondot@med.u-toyama.ac.jp

doi : $10.3191 /$ thermalmed.23.113

(C) 2007 Japanese Society for Thermal Medicine 
ultrasound with or without microbubbles also exhibited promising results. Membrane damage is pivotal to biological effects, and using ultrasound to modify or affect cell membranes can either promote or inhibit desired effects. In summary, it is concluded that ultrasound has the potential to help develop useful methods which can be utilized in therapies which require apoptosis induction, gene introduction into cells, alterations in gene regulation, and drug-activation.

Key Words : drug activation, apoptosis, gene transfer, gene expression, microbubble

\section{Introduction}

The biophysical modes of ultrasound action are classified as thermal, cavitational, and non-thermal non-cavitational effects. Cavitation can be classified into two types, stable (non-inertial) and transient (inertial). Stable cavities are bubbles which oscillate radially at their equilibrium resonance size and persist for many acoustic cycles. Transient cavities, however, exist for only one or two acoustic cycles and will expand to two to three times their resonance size before collapsing violently during a single compression half cycle. The temperature and pressure in an imploding cavity may be in the order of several thousand degrees $\mathrm{K}$ and hundreds of atmospheres, respectively. In water, this leads to the formation of hydroxyl radicals and hydrogen atoms due to the thermal decomposition of water molecules. Moreover, the collapse of a bubble influences its local neighborhood by generating hydrodynamic and mechanical effects such as the formation of "micro jets", shock waves, and shear stresses").

Recently, therapeutic applications of ultrasound which utilize thermal effects for tissue ablation have been studied extensively and used clinically. In addition to the thermal effects of ultrasound, the therapeutic use of non-thermal effects such as acoustic cavitation is also becoming an interesting subject for researchers ${ }^{2,3)}$. The effects of cavitation combined with hyperthermia ${ }^{4)}$, hypotonia ${ }^{5)}$ and local anesthetics which are membrane-modulating agents ${ }^{6,7)}$ were examined, and studies indicated that this type of protocol could lead to the effective enhancement of biological effects. Cellular membrane damage caused by sonomechanical effects due to cavitation is pivotal in events leading to cell death, and in introducing macromolecules into cells by taking advantage of cellular membrane damage and repair mechanisms in damaged cells. Below, current data is reviewed concerning 1) the activation of an anticancer agent, doxorubicin $(\mathrm{DOX}) ; 2$ ) an apoptosis mechanism and its optimal induction with minimal cell lysis; 3) enhancement of apoptosis in the presence of an echo-contrast agent; 4) enhancement of apoptosis combined with a free radical generator; 5) changes in gene-expression and the mechanism involved; and 6) utilization of ultrasound for gene transfer, and enhancement of liposome-mediated gene transfer by low intensity ultrasound.

\section{1) Enhancement of cell killing and apoptosis due to DOX combined with ultrasound.}

An additive or enhanced effect of ultrasound and anti-cancer drugs has been reported ${ }^{2)}$. Among anti-cancer drugs, DOX is one of the most potent drugs whose anti-cancer effect is enhanced by ultrasound. Although an increased incorporation of the drug in response to ultrasound was reported, the detailed mechanism of this enhancement has not been completely clarified.

Recently, low-intensity pulsed ultrasound was utilized to 1) validate the enhancement effect on cell 
killing, 2) evaluate the enhancement of apoptosis induction, 3) examine cavitational and non-cavitational effects, and 4) investigate the involvement of sonoporation.

Cell killing and induction of apoptosis were investigated in human lymphoma U937 cells. When cells were exposed to ultrasound at power levels above $0.3 \mathrm{~W} / \mathrm{cm}^{2}$, where hydroxyl radical formation can be used to indicate acoustic cavitation, a synergistic enhancement in cell killing and an additive enhancement in apoptosis induction were observed. No enhancement was observed at an intensity of 0.2 $\mathrm{W} / \mathrm{cm}^{2}$. The production of hydroxyl radicals was enhanced by the presence of DOX at ultrasound intensities over $0.3 \mathrm{~W} / \mathrm{cm}^{2}$. Incorporation of DOX into cells was found to increase by $13 \%$ if DOX was present with ultrasound exposure (vs. the DOX alone group) at an intensity of $0.5 \mathrm{~W} / \mathrm{cm}^{2}$. The incorporation of fluorescein isothiocyanate (FITC)-dextran was used as an indicator of sonoporation. The data obtained in this study suggests that the mechanisms involved in this enhancement are, 1) an increase in the incorporation of DOX resulting from sonoporation, and 2) an increase in cavitational activity caused by DOX (Fig. 1). In addition, when the cell suspensions containing DOX were exposed to ultrasound in the presence of echo-contrast microbubbles (Levovist) to enhance cavitational activity, a further enhancement of cell killing and apoptosis was observed. Microbubbles are useful in the therapeutic application of ultrasound combined with $\mathrm{DOX}^{8)}$.

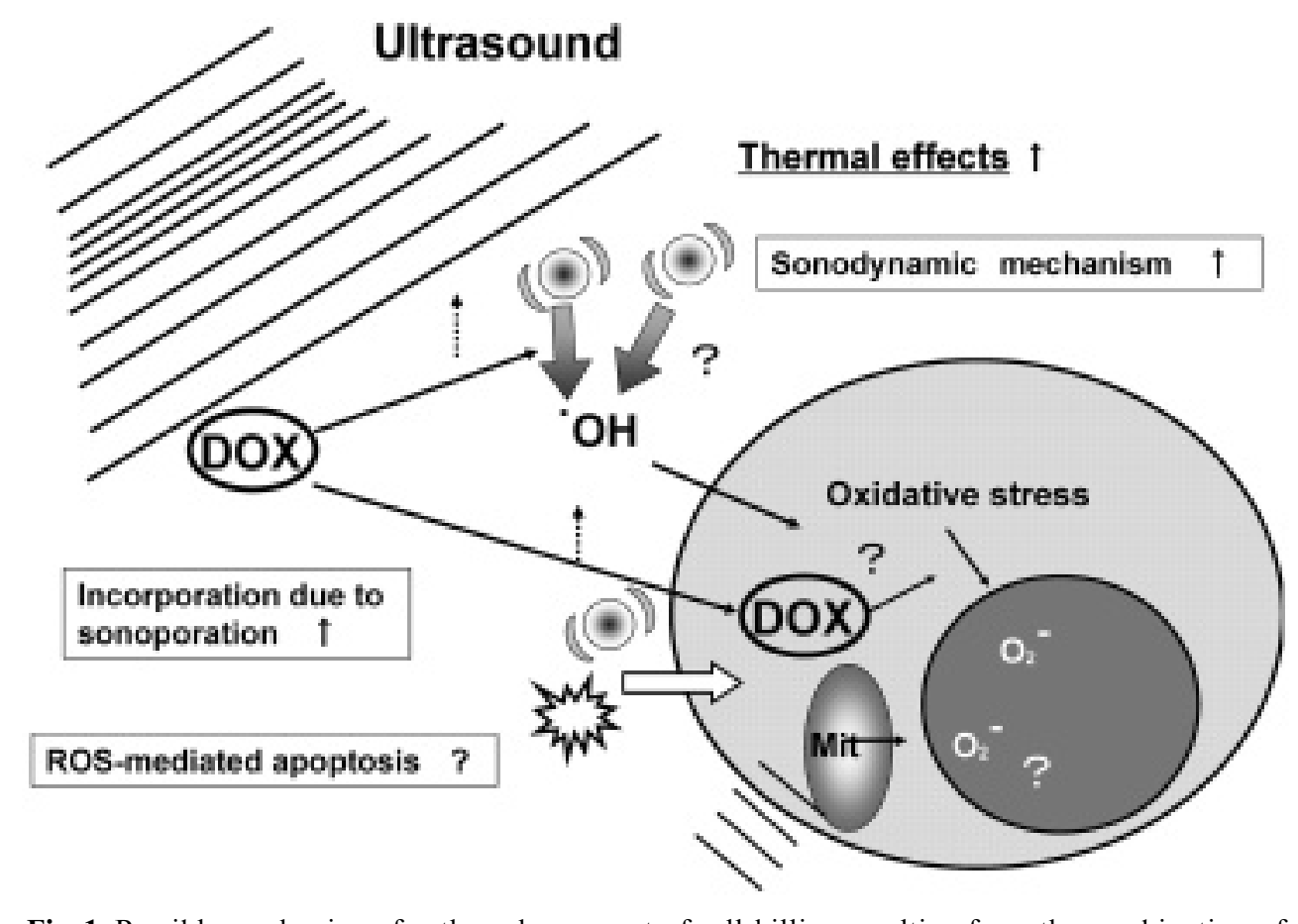

Fig. 1. Possible mechanisms for the enhancement of cell killing resulting from the combination of ultrasound and doxorubicin (DOX). ROS : reactive oxygen species; Mit: mitochondria.

2) Apoptosis induction by ultrasound. Optimal apoptosis induction with minimal cell lysis.

Apoptosis has uniquely defined morphological and molecular characteristics, and is a mechanism which leads to the destruction of cells. This mechanism plays an important role in a variety of 
biological events, including the surveillance of tumors and other malfunctioning cells. Human lymphoma U937 cells were exposed to continuous $1 \mathrm{MHz}$ ultrasound, a level used for therapy, and apoptosis and its endpoints were examined. Significant superoxide formation, a decrease in mitochondria membrane potential (MMP), increased activity of caspase-3, and DNA fragmentation were found in apoptotic cells induced by ultrasound ${ }^{9)}$.

When the effects of dissolved gases on free radical formation due to inertial cavitation were investigated with electron paramagnetic resonance (EPR) spin trapping, the formation of hydroxyl radicals and hydrogen atoms was observed in solutions saturated with mono- and di-atomic gases, but not in solutions saturated with triatomic gases. Apoptosis induced by ultrasound was seen to be influenced by the gases dissolved in the medium. Evidence of mitochondria-caspase dependent apoptosis in U937 cells induced by $1 \mathrm{MHz}$ ultrasound in the presence of various gases, but not by triatomic gases was obtained. These results suggest that ultrasound-induced apoptosis apparently resulted from inertial cavitation, although quantities of extracellular free radicals did not influence the outcome ${ }^{9)}$. In another study, a role for intracellular reactive oxygen species and calcium ions in apoptosis was shown ${ }^{10)}$.

To determine the optimal conditions for ultrasound-induced apoptosis which could be useful in cancer therapy, low intensity pulsed ultrasound was applied to U937 cells in vitro. Cells were then incubated for different time intervals before measuring the percent of apoptotic cells. Apoptosis was scored by DNA fragmentation and phosphatidylserine externalization. MMP decreases were determined with flow cytometry. Optimal apoptosis $(\sim 70 \%)$ with minimal lysis was attained with $1 \mathrm{MHz}$ ultrasound $\left(0.3 \mathrm{~W} / \mathrm{cm}^{2}, 10 \%\right.$ duty factor at $100 \mathrm{~Hz}$ for $\left.1 \mathrm{~min}\right)$ at $12 \mathrm{hrs}$ after exposure. A lack of ultrasound-induced free radicals and the absence of up-regulation of heme oxygenase (HO)-1, an intracellular oxidative stress marker, suggest that sonomechanical, and not sonochemical, effects were the primary mechanism involved ${ }^{11)}$.

\section{3) Enhancement of apoptosis induced by low intensity ultrasound in the presence of echo-contrast agents.}

To verify the effect of an echo-contrast agent on ultrasound induced apoptosis, leukemia cell lines (Jurkat, Molt-4 and U937) were exposed to ultrasound at intensities previously shown to induce optimal apoptosis with or without Levovist. The results showed that a loss of viability and an increase in apoptosis could be seen in all three cell lines, with Molt-4 cells exhibiting the highest level of apoptosis. These results were obtained by measuring viability and using a DNA fragmentation assay. This finding was supported by a corresponding increase of cells with low MMP, high superoxide production, increased intracellular calcium concentrations, and DNA double strand breaks after exposure. The optimal ultrasound conditions were $0.3 \mathrm{~W} / \mathrm{cm}^{2}, 1 \mathrm{MHz}, 10 \%$ duty factor, and a pulse frequency of $100 \mathrm{~Hz}$. In the presence of Levovist, an apparent shift of maximal cell killing induction to $0.2 \mathrm{~W} / \mathrm{cm}^{2}$ was observed ${ }^{12)}$. While these results confirmed previous findings for ultrasound-induced apoptosis, they also suggest that the use of an enhancing factor, such as the addition of echo-contrast agents, may be useful in cancer therapy when a much lower intensity of ultrasound is desirable (Fig. 2) ${ }^{13)}$. 


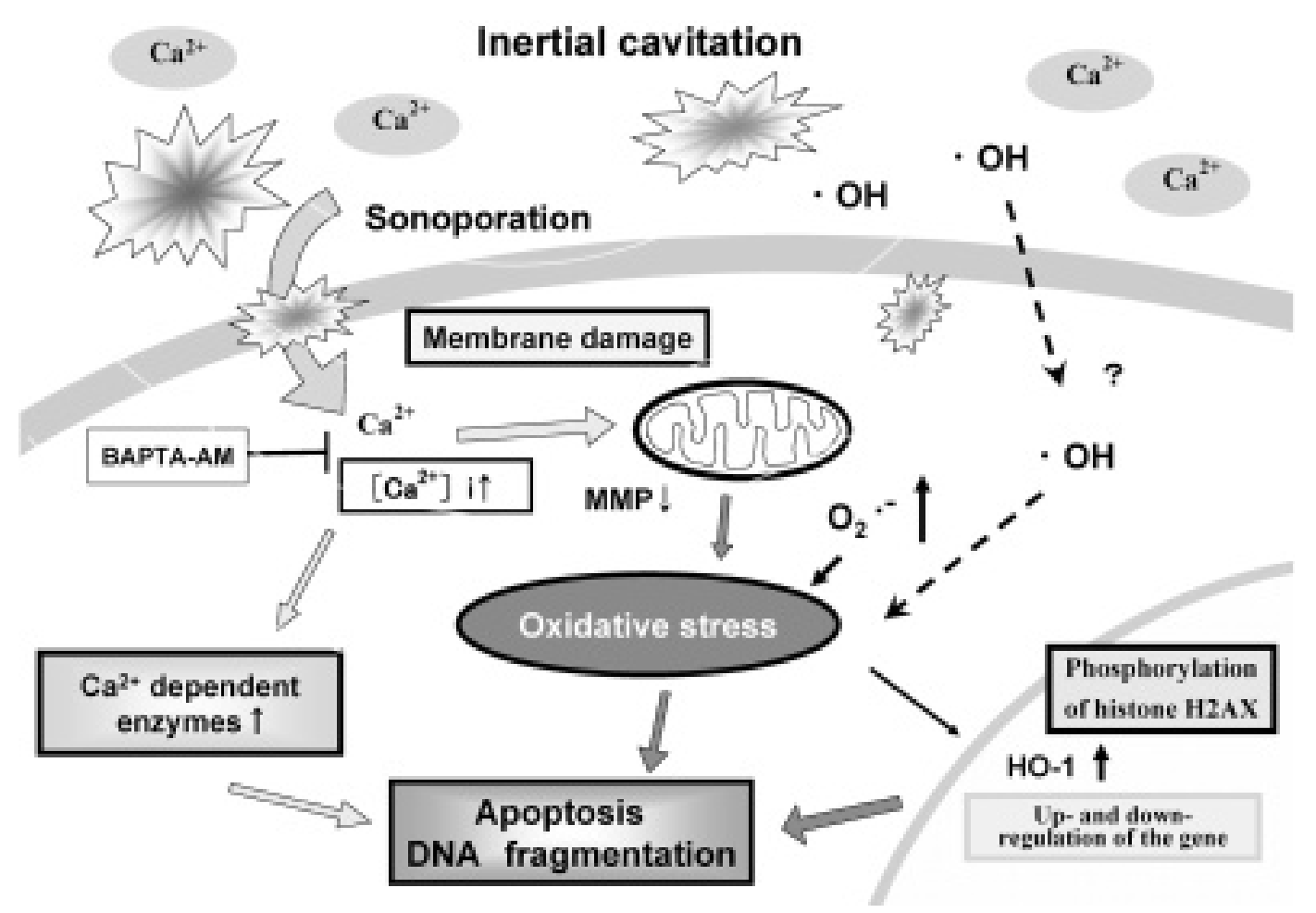

Fig. 2. Possible mechanisms of apoptosis and alterations in gene expression induced by ultrasound. MMP : mitochondrial transmembrane potential; HO-1: heme oxygenase-1.

\section{4) Enhancement of apoptosis when combined with a free radical generator.}

To determine the effect of 2,2'-azobis (2-amidinopropane) dihydrochloride (AAPH), a temperature-dependent free radical generator, on ultrasound-induced cell killing, human leukemia U937 cells were incubated at various temperatures $\left(25,37\right.$ and $\left.40^{\circ} \mathrm{C}\right)$ for $1 \mathrm{~min}$ in air-saturated PBS containing $50 \mathrm{mM}$ AAPH before being exposed to $1 \mathrm{MHz}$ ultrasound for $1 \mathrm{~min}$ at an intensity of $2.0 \mathrm{~W} / \mathrm{cm}^{2}$ under thermally controlled conditions. Cell viability was determined with the trypan blue dye exclusion test immediately after ultrasound exposure. Apoptosis was measured with flow cytometry after a $6 \mathrm{hrs}$ post-exposure incubation. Free radicals generated by AAPH or ultrasound or both were also investigated using EPR-spin trapping. The results showed that ultrasound-induced cell lysis and apoptosis were enhanced in the presence of AAPH regardless of the temperature at the time of sonication. At $40^{\circ} \mathrm{C}$, ultrasound alone induced increased cell killing, while AAPH alone was capable of inducing significant but minimal apoptosis at this temperature. Although free radical concentrations increased with the combined treatment, this free radical increase did not correlate well with cell killing. The mechanism of enhancement points to an increased uptake of the agent during ultrasound exposure rather than a potentiation by AAPH. These findings suggest a potential role for temperature-dependent free radical generators in cancer therapy using therapeutic ultrasound ${ }^{14)}$.

Studies on interactions between ultrasound and chemicals are of interest because of potential therapeutic applications involving the activation and control of drugs by extracorporeal physical factors such as ultrasound. Recently, it was shown that nitric oxide could be released from its caged compound 
by ultrasound ${ }^{15)}$, and also that there are sonodynamic effects on rhodamine derivatives ${ }^{16)}$.

\section{5) Change of gene-expression and a possible mechanism.}

DNA microarray expression profiling offers a tremendous potential for characterizing gene expression patterns which can be used to identify specifically expressed genes which are induced by physical and chemical stresses. A study was undertaken to investigate the changes in gene expression in human cultured cells exposed to $1 \mathrm{MHz}$ ultrasound. Monoatomic Ar gas was used to amplify inertial cavitation, while triatomic $\mathrm{N}_{2} \mathrm{O}$ gas was used to eliminate inertial cavitation. Free radical formation and hemolysis were utilized as endpoints for inertial cavitation. Although the formation of hydroxyl radicals and hydrogen atoms were observed along with hemolysis in aqueous solutions exposed at an intensity of $4.9 \mathrm{~W} / \mathrm{cm}^{2}$ in the presence of Ar, no free radical formation or hemolysis were observed in the presence of $\mathrm{N}_{2} \mathrm{O}$. Gene expression in the remaining unlysed human lymphoma cells (U937) at 6 hrs after sonication was examined by DNA microarray profiling to identify genes whose expression was altered in response to ultrasound exposure. Genes were considered up- or down-regulated if their average fold change was 2.0 or greater, and using this criteria, only the keratin gene was identified as a down-regulated gene in ultrasound-exposed cells in the presence of $\mathrm{N}_{2} \mathrm{O}$. However, five genes were identified as up-regulated and two genes as down-regulated in the presence of Ar. Among four genes, except EST, which showed alterations in gene expression, heme oxygenase-1 (HO-1) and v-jun were confirmed to be up-regulated, and cathepsin $G$ and v-myb were shown to be down-regulated using a semi-quantitative polymerase chain reaction assay (PCR). Gene expression of HO-1 increased by a factor of 6.6 according to the DNA array assay, and by a factor of 4.0 with PCR. These results indicate that inertial cavitation increased the expression of genes responsive to oxidative stress in exposed cells, but non-inertial cavitation cause a minimal effect on gene expression ${ }^{17}$. Subsequently, another study was undertaken to confirm HO-1 induction by ultrasound and to elucidate the mechanism involved. After exposure of U937 cells to $1 \mathrm{MHz}$ continuous waves, gene profiling with DNA microarray analysis, mRNA expression measured with real-time semi-quantitative PCR, and protein expression measured with western blotting were studied. DNA microarray analysis confirmed the HO-1 induction seen in U937 cells after exposure to ultrasound, and further identified one up-regulated [PCTAIRE protein kinase 1 transcript variant 2 (PCTK1-V2)] and two down-regulated [leukemia inhibitory factor receptor (LIFR) and chemokine ligand 10 (CXCL10)] genes. When U937 cells were exposed for $1 \mathrm{~min}$, HO-1 induction, examined with real-time semi-quantitative PCR and western blotting, was observed to occur at intensities greater than the cavitational threshold. When a potent antioxidant, N-acetyl-L-cysteine was added to the culture medium before or after ultrasound exposure, this induction was attenuated, indicating that reactive oxygen species were involved in HO-1 induction. A decrease in MMP and the generation of superoxide anion radicals was also observed in cells exposed to ultrasound. It appears that the enhancement of HO-1 expression may result primarily from increased intracellular oxidative stress, and secondarily from sonomechanical effects arising from ultrasonic cavitation ${ }^{18)}$.

Recently, gene expression in human leukemia Molt-4 cells treated with non-thermal low intensity pulsed ultrasound was examined. A frequency of about $24 \%$ apoptosis with minimal cell lysis was observed at $6 \mathrm{hrs}$ after treatment with pulsed ultrasound at an intensity of $0.3 \mathrm{~W} / \mathrm{cm}^{2}$. Of the 
approximately 16,600 genes analyzed, BCL2-associated athanogene 3 (BAG3), a DnaJ (Hsp40) homolog, subfamily B, member 1 (DNAJB1), heat shock $70 \mathrm{kDa}$ protein 1B (HSPA1B), and heat shock $70 \mathrm{kDa}$ protein 6 (HSPA6) showed increased levels of expression, while isopentenyl-diphosphate delta isomerase (IDI1) and 3-hydroxy-3-methylglutaryl-coenzyme A synthase 1 (HMGCS1) showed decreased levels 3 hrs after the ultrasound treatment. The expression levels of these six genes were confirmed by real-time semi-quantitative PCR. This appears to be the first report, using DNA microarray analysis, of genes which are differentially expressed in response to apoptosis induced by non-thermal low intensity pulsed ultrasound in human leukemia cells ${ }^{19)}$.

\section{6) Usefulness of ultrasound for gene transfer.}

Recently, a review of ultrasound-mediated gene transfection with or without microbubbles was published $^{20)}$ (Fig. 3), and the effects of ultrasound on liposome-mediated gene transfer are discussed here. It has been shown that some types of liposomes, usually containing cationic lipids, are useful tools for gene introduction into cells. To investigate the effect of ultrasound on liposome-mediated transfection, three types of liposomes (designated L1, L2 and L3, in their order of increasing transfection efficiency) containing DC-6-14, DOPE, and/or cholesterol at varying ratios, were used in this study. HeLa cells were treated with liposome-DNA complexes containing a luciferase gene for $2 \mathrm{hrs}$ before sonication. The optimal acoustic setting for lipofection enhancement was determined to be $0.5 \mathrm{~W} / \mathrm{cm}^{2}, 1 \mathrm{MHz}$ continuous waves for $1 \mathrm{~min}$, and was above the threshold for inertial cavitation based on the EPR

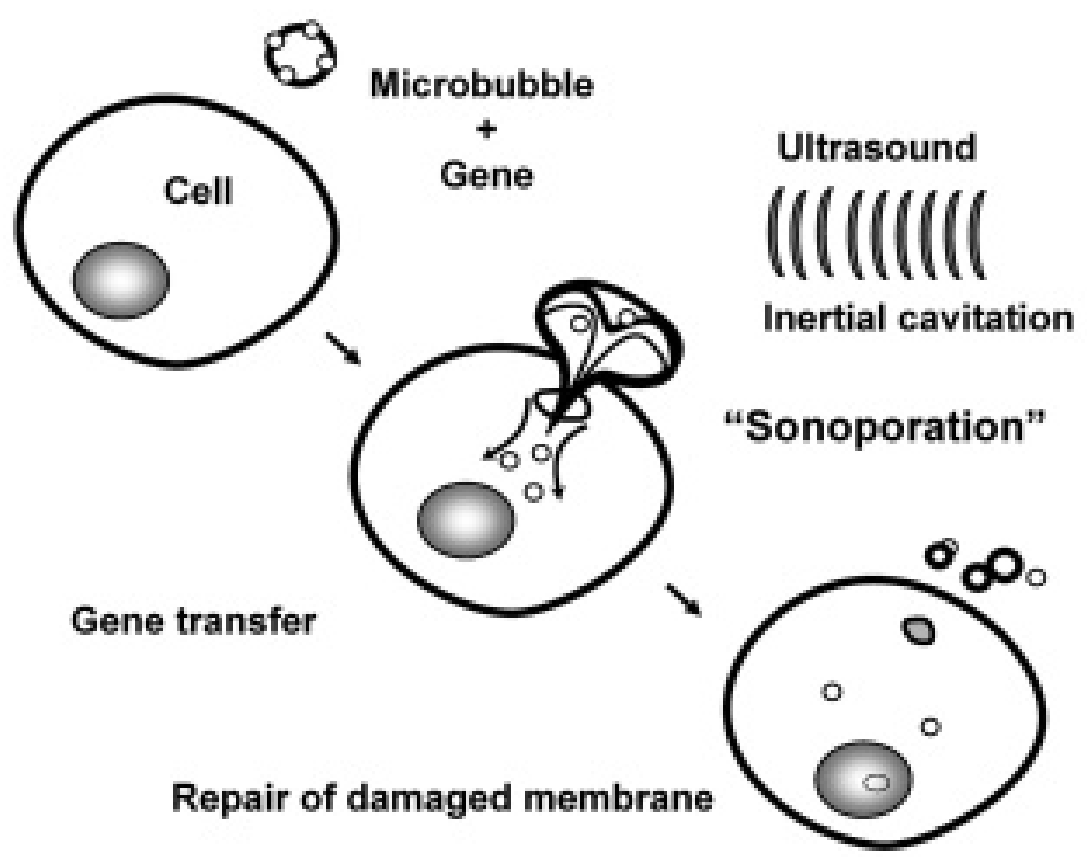

Fig. 3. Gene transfer by "sonoporation". Ultrasound at certain intensity levels creates inertial cavitation that can lead to membrane damage from microjets. Microjets cause transient pore formation in the membrane. During the rapid resealing period of these induced pores, genes and high molecular weight chemicals can be introduced into cells. 
detection of free radicals. Luciferase expression $24 \mathrm{hrs}$ after the treatment was significantly increased by sonication by 2.4 fold with L1, and 1.7 fold with L2. However, with L3, which showed the highest level of expression among the liposomes without sonication, a significant but minimal enhancement was observed when sonication was carried out $15 \mathrm{~min}$ after the DNA-L3 treatment, suggesting that the efficiency of the individual liposome also determines the proper timing for ultrasound exposure. A 2 hrs pre-exposure incubation with liposome-DNA complexes for L1 and L2 (30 min for L3) was required to attain transfection enhancement, suggesting that ultrasound works to enhance transfection only after cells had already begun DNA uptake ${ }^{21)}$. Liposomes combined with specific antibodies have been designed for molecularly targeted therapy of drug and/or gene delivery. Recently, optimal acoustic conditions for ultrasound-mediated gene transfection were studied in a variety of cancer cell lines, and it was found that optimum ultrasound conditions were $1 \mathrm{MHz}$ at $1.5 \mathrm{~W} / \mathrm{cm}^{2}$ with a pulse frequency of $0.5 \mathrm{~Hz}$, and with a duty factor of $50 \%$. These results showed that the transfection rate increased with the number of pulses, and peaked between 10 and 15 pulses $^{22)}$.

\section{Summary and implications}

Biological effects which may be potentially useful in the treatment of diseases such as cancer and immune disorders are being studied. Apoptosis induction by ultrasound may provide a method to induce a direct and rapid treatment targeting tumors. Systemic or local sonotransfection might also provide a safe and effective regimen for gene therapy in treating certain disorders. Gene regulation of target cells may also be modified by altering cellular responses to treatment, for example, by increasing the sensitivity of diseased cells. With low intensity ultrasound, where bulk temperature increases are not significant and free radical production is minimal, mechanical effects could also be useful in therapeutic applications. The term "sonomechanical effects" is used here in contrast to "sonodynamic effects" and "thermal effects". Sonodynamic effects are usually associated with free radical production, while thermal effects are associated with bulk temperature rises which are used for hyperthermia. On the other hand, the mechanical effects due to low intensity ultrasound are predominantly generated by cavitation, inertial or non-inertial. Also the role of engineered microbubbles in attaining a well defined and modulated therapeutic effect will be crucial in the actual application of low-intensity ultrasound for therapy.

\section{Acknowledgements}

This study is supported in part by Grants in Aid for Scientific Research (B) (17310032) from the Ministry of Education, Culture, Sports, Sciences and Technology, as part of the Research and Development Committee Program of the Japan Society of Ultrasonics in Medicine, and the academic exchange and cooperation between Graduate School of Medicine and Pharmaceutical Sciences, University of Toyama, Japan and Tomsk Cancer Research Institute, Russian Federation. 


\section{References}

1) Riesz P., Kondo T. : Free radical formation induced by ultrasound and its biological implications. Free Radic Biol Med, $13: 247-270,1992$.

2) Feril L.B.Jr., Kondo T., Umemura S-i., Tachibana K., Manalo A.H., Riesz P. : Sound waves and anti-neoplastic drugs : The possibility of an enhanced combined anticancer therapy. J Med Ultrasonics, 29: 173-187, 2002.

3) Feril L.B.Jr., Kondo T. : Biological effects of low intensity ultrasound: the mechanism involved, and its implications on therapy and on biosafety of ultrasound. J Radiat Res, 45 : 479-489, 2004.

4) Feril L.B.Jr., Kondo T., Zhao Q.L., Ogawa R. : Enhancement of hyperthermia-induced apoptosis by non-thermal effects of ultrasound. Cancer Lett, 178: 63-70, 2002.

5) Feril L.B.Jr., Kondo T., Takaya K., Riesz P. : Enhancement of ultrasound-induced apoptosis and cell lysis by a hypotonic medium. Int J Radiat Biol, 80: 165-175, 2004.

6) Nozaki T., Ogawa R., Feril L.B.Jr., Kagiya G., Fuse H., Kondo T.: Enhancement of ultrasound-mediated gene transfection by membrane modification. J Gene Med, 5 : 1046-1055, 2003.

7) Ogawa R., Kagiya G., Feril L.B.Jr., Nakaya N., Nozaki T., Fuse H., Kondo T.: Ultrasound mediated intravesical transfection enhanced by treatment with lidocaine or heat. J Urol, 172: 1469-1473, 2004.

8) Yoshida T., Kondo T., Ogawa R., Feril L.B.Jr., Zhao Q.L., Watanabe A., Tsukada K. : Combination of doxorubicin and low-intensity ultrasound causes a synergistic enhancement in cell killing and an additive enhancement in apoptosis induction in human lymphoma U937 cells. Cancer Chemother Pharmacol, (in press).

9) Honda H., Zhao Q.L., Kondo T. : Effects of dissolved gases and an echo contrast agent on apoptosis induced by ultrasound and its mechanism via the mitochondria-caspase pathway. Ultrasound Med Biol, 28 : 673-682, 2002.

10) Honda H., Kondo T., Zhao Q.L., Feril L.B.Jr., Kitagawa H. : Role of intracellular calcium ions and reactive oxygen species in apoptosis induced by ultrasound. Ultrasound Med Biol, 30 : 683-692, 2004.

11) Feril L.B.Jr., Kondo T., Cui Z.G., Tabuchi Y., Zhao Q.L., Ando H., Misaki T., Yoshikawa H., Umemura S-i. : Apoptosis induced by the sonomechanical effects of low intensity pulsed ultrasound in a human leukemia cell line. Cancer Lett, $221:$ 145-152, 2005.

12) Ando H., Feril L.B.Jr., Kondo T., Tabuchi Y., Zhao Q.L., Cui Z.G., Umemura S-i., Yoshikawa H., Misaki T. : An echo-contrast agent, Levovist, lowers the ultrasound intensity required to induce apoptosis of human leukemia cells. Cancer Lett, 242: 37-45, 2006.

13) Feril L.B.Jr., Kondo T., Zhao Q.L., Ogawa R., Tachibana K., Kudo N., Fujimoto S., Nakamura S. : Enhancement of ultrasound-induced apoptosis and cell lysis by echo contrast agents. Ultrasound Med Biol, 29: 331-337, 2003.

14) Feril L.B.Jr., Tsuda Y., Kondo T., Zhao Q.L., Ogawa R., Cui Z.G., Tsukada K., Riesz P. : Ultrasound-induced killing of monocytic U937 cells enhanced by 2, 2'-azobis (2-amidinopropane) dihydrochloride. Cancer Sci, 95 : 181-185, 2004.

15) Feril L.B.Jr., Kondo T.: Ultrasound liberates nitric oxide (NO) from the caged NO compound N, N-bis (carboxymethyl)-N, N'-dinitroso-p-phenylenediamine sodium salt. Ultrason Sonochem, 13: 397-400, 2006.

16) Hiraoka W., Honda H., Feril L.B.Jr., Kudo N., Kondo T.: Comparison between sonodynamic effect and photodynamic effect with photosensitizers on free radical formation and cell killing. Ultrason Sonochem, 13: 535-542, 2006.

17) Tabuchi Y., Kondo T., Ogawa R., Mori H. : DNA microarray analyses of genes elicited by ultrasound in human U937 cells. Biochem Biophys Res Commun, 290 : 498-503, 2002.

18) Kagiya G., Tabuchi Y., Feril L.B.Jr., Ogawa R., Zhao Q.L., Kudo N., Hiraoka W., Tachibana K., Umemura S-i., Kondo T. : Confirmation of enhanced expression of heme oxygenase-1 gene induced by ultrasound and its mechanism : Analysis by cDNA microarray system, real time quantitiative PCR, and western blotting. J Med Ultrasonics, 33: 3-10, 2006. 
Thermal Med. 23 [3] : 2007

19) Tabuchi Y., Ando H., Takasaki I., Zhao Q.L., Ogawa R., Kudo N., Tachibana K., Kondo T. : Identification of genes responsive to low intensity pulsed ultrasound in a human leukemia cell line Molt-4. Cancer Lett, 246 : 149-156, 2007.

20) Nozaki T., Ogawa R., Watanabe A., Nishio R., Fuse H., Kondo T. : Ultrasound- mediated gene transfection : Problems to be solved and future possibilities. J Med Ultrasound, 33 : 135-144, 2006.

21) Feril L.B.Jr., Ogawa R., Kobayashi H., Kikuchi H., Kondo T.: Ultrasound enhances liposome-mediated gene trasfection. Ultrason Sonochem, 12 : 489-493, 2005.

22) Feril L.B.Jr., Ogawa R., Tachibana K., Kondo T. : Optimized ultrasound-mediated gene transfection in cancer cells. Cancer Sci, 97 : 111-114, 2006. 